\title{
Exploring Heterogeneity in Li Battery Electrodes using FIB-SEM Integrated with Raman and TOF-SIMS
}

\author{
Dean J. Miller ${ }^{1}$, Dave Zapotok ${ }^{1}$, Jon Hiller ${ }^{1}$, Zdenek Juracka ${ }^{1}$, Ute Schmidt ${ }^{2}$, Tavis Ezell ${ }^{2}$, Nancy \\ Senabulya $^{3}$, Bobby Kerns ${ }^{3}$, Marco-Tulio Fonseca Rodrigues ${ }^{4}$, Victor A. Maroni ${ }^{4}$ and Daniel P. \\ Abraham $^{4}$ \\ 1. TESCAN USA, Warrendale, PA, USA. \\ 2. WITec GmbH, Ulm, Germany. \\ 3. Michigan Center for Materials Characterization, University of Michigan, Ann Arbor, MI, USA. \\ 4. Chemical Sciences \& Engineering Division, Argonne National Laboratory, Argonne, IL, USA.
}

During electrochemical cycling of Li-ion batteries, microstructural changes can lead to a loss of energy and power, especially under rapid charging. Changes in performance are often nonuniform as a result of inhomogeneous degradation in which some local regions in battery electrodes may experience more significant degradation than other regions. This inhomogeneous deterioration has been studied using a variety of approaches including, for example, X-ray diffraction [1], transmission X-ray microscopy and tomography [2,3], and transmission electron microscopy [4,5]. FIB-SEM provides a powerful, complementary way to explore microstructure by providing the capability to look at a material in crosssection, especially in conjunction with other techniques such as Raman that can reveal subtle variations in composition and chemistry [6].

In this work, the inhomogeneous deterioration of microstructure in Li-ion battery electrodes subjected to fast charging and long-term cycling was studied using FIB-SEM integrated with Raman spectroscopy. Complementary measurements using TOF-SIMS integrated into FIB-SEM were also used to evaluate local degradation. Combined with FIB-SEM, these techniques can be applied to electrode cross-sections to provide unique information from regions across the thickness of the electrode. In particular, a confocal Raman system integrated with SEM or FIB-SEM provides sufficient resolution to evaluate variations even within single particles that can be correlated with structure at the SEM scale.

Figure 1a shows a Raman image from a cathode electrode cross section prepared by FIB-SEM. The electrode is from an NCM523/graphite full cell subjected to $400,3.0-4.4 \mathrm{~V}$ cycles at $30^{\circ} \mathrm{C}$ resulting in a $40 \%$ loss of capacity. In this Raman image, individual cathode oxide particles and the supporting carbon network are delineated based on the Raman spectrum at each point in the image. Representative spectra for the cathode and carbon regions are shown in Fig. 1b. Already at this level of imaging some variations in the cathode particles from region to region can be observed. A more detailed view of a single cathode particle is shown in the Raman image of Fig. 2a. Here, local differences in structure within a single particle are observed with corresponding Raman spectra shown in Fig. 2c. The envelope of bands in the range of 400-750 $\mathrm{cm}^{-1}$ (marked as region 1 in Fig. 2c) is associated with metal-oxygen (M-O) vibrations and the shape of this envelope changes with lithiation, more details of which are described elsewhere [6,7]. This Raman data reveals local variations in structure and lithiation even at the single particle level. Similar variations in local chemistry and structure can be observed by TOF-SIMS, as shown in Fig. 2b which shows the heterogeneous distribution of $\mathrm{Li}$ in a single particle. In addition to variations in the $\mathrm{M}$ $\mathrm{O}$ vibrations, some regions within the particle exhibit Raman spectra that also show signatures of carbon (region 2 in Fig. 2c). The presence of carbon in the interior of the particles is most likely related to the penetration of the $\mathrm{LiPF}_{6}$-carbonate electrolyte along grain boundaries that separate upon cycling [8]. Measurements on pristine, uncycled particles do not show evidence of carbon in the interior. 
The integration of FIB-SEM with Raman and TOF-SIMS provides the opportunity to explore electrode inhomogeneity in a unique way. These results reveal heterogeneous deterioration of structure in Li-ion oxide cathode particles that can contribute to reduced performance and life of lithium-ion cells.

References:

[1] J Liu, et al., J. Phys. Chem. Lett. 1 (2010), p. 2120.

[2] WE Gent, et al., Adv. Mater. 28 (2016), p. 6631.

[3] P Pietsch and V Wood, Annu. Rev. Mater. Res. 47 (2017), p. 451.

[4] PJ Phillips et al., Adv. Energy Mater. 5 (2015), p. 1501252.

[5] ME Holtz et al., Nano Lett. 14 (2014), p. 1453.

[6] JA Gilbert et al., Adv. Mater. Interfaces 5 (2018), p. 1701447.

[7] R Baddour-Hadjean and J-P Pereira-Ramos, Chem. Rev. 110 (2010), p. 1278.

[8] DJ Miller et al., Adv. Energy Mater. 3 (2013), p. 1098.
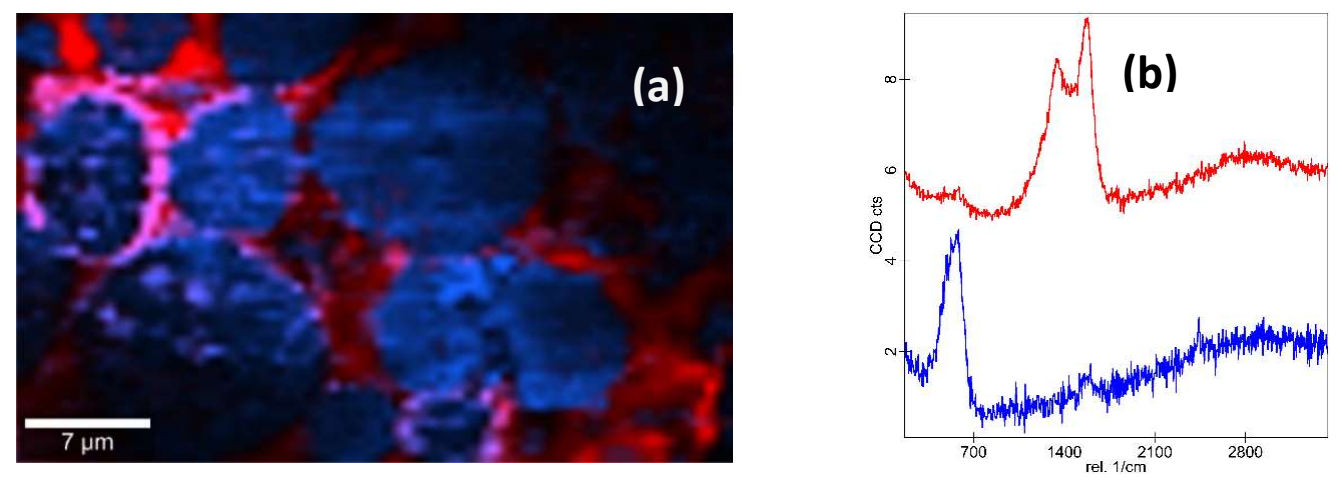

Figure 1. (a) Raman image of an Li-ion battery cathode electrode cross-section with (b) corresponding spectra. Colors in image (a) correspond to spectra of the same color in (b).
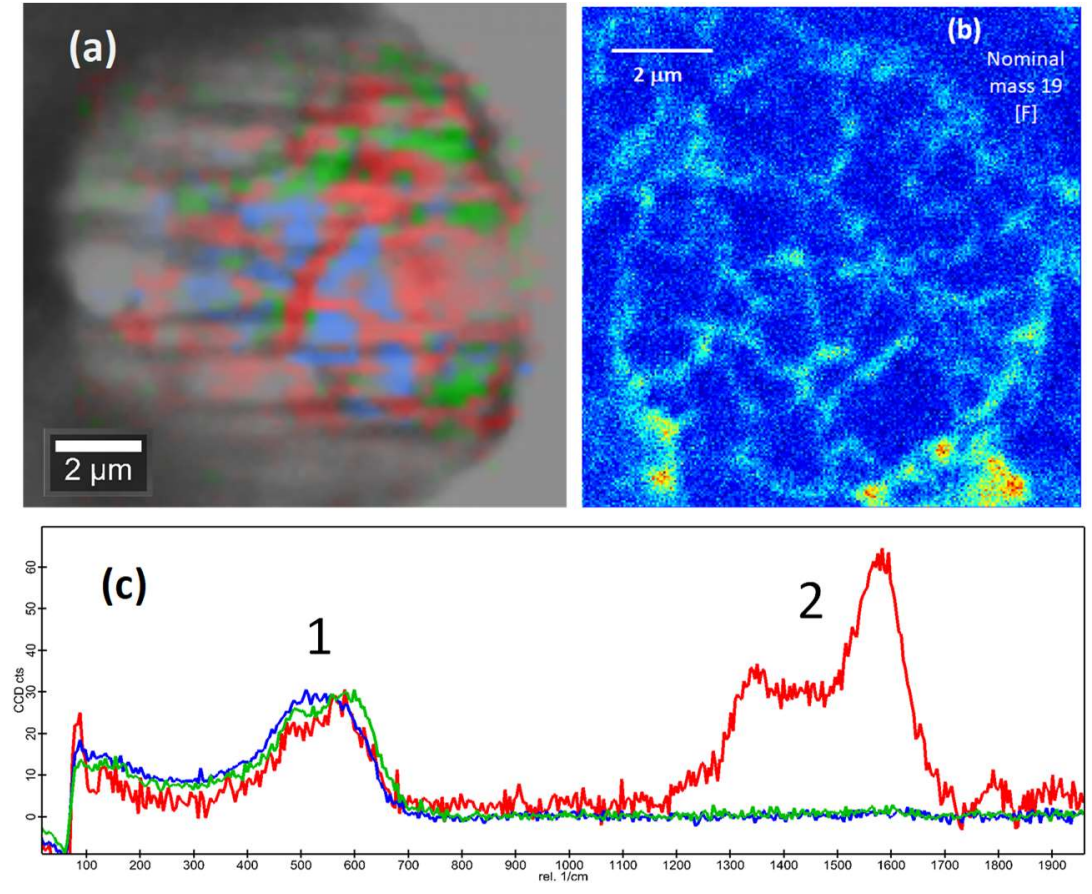

Figure 2. (a) Raman/optical image and (b) top projection SIMS image for nominal mass 7 (Li) from single NCM cathode particles. Colors in image (a) correspond to spectra of the same color in (c). 